\title{
Féeries
}

Études sur le conte merveilleux, XVII $-\mathrm{XIXe}$ siècle

\section{Marina Warner, From the Beast to the Blonde. On Fairy Tales and their Tellers}

The Noonday Press, Farrar, Straus and Giroux, New York 1999 ( $1^{\text {re }}$ éd. pour Chatto \& Windus, London, 1994), $463 \mathrm{p}$.

Jean-Paul Sermain

\section{OpenEdition}

Journals

Édition électronique

URL : http://journals.openedition.org/feeries/90

DOI : $10.4000 /$ feeries.90

ISSN : 1957-7753

Éditeur

UGA Éditions/Université Grenoble Alpes

Édition imprimée

Date de publication : 1 février 2004

Pagination : 205-209

ISSN : 1766-2842

Référence électronique

Jean-Paul Sermain, " Marina Warner, From the Beast to the Blonde. On Fairy Tales and their Tellers », Féeries [En ligne], 1 | 2004, mis en ligne le 29 janvier 2007, consulté le 24 septembre 2020. URL : http:// journals.openedition.org/feeries/90 ; DOI : https://doi.org/10.4000/feeries.90

Ce document a été généré automatiquement le 24 septembre 2020

(c) Féeries 


\section{Marina Warner, From the Beast to the Blonde. On Fairy Tales and their Tellers}

The Noonday Press, Farrar, Straus and Giroux, New York 1999 ( $1^{\text {re }}$ éd. pour Chatto \& Windus, London, 1994), $463 \mathrm{p}$.

Jean-Paul Sermain

Pourquoi traiter d'un livre publié il y a dix ans ? Parce qu'il reste peu connu du public français (en attente d'une traduction) et qu'il est la contribution la plus importante consacrée au conte de fées littéraire depuis l'ouvrage de Raymonde Robert, avec une plus grande largeur de vue encore, par contraste avec les choix de la critique française. Celle-ci, sans doute par hasard, a porté son attention sur un seul auteur, Mme d'Aulnoy : Anne Defrance a défriché un domaine jusqu'alors inexploré, attentive à la fois aux agencements discursifs et aux implications symboliques des contes; Jean Mainil s'est surtout attaché aux effets de l'emboîtement des récits en prenant pour ligne directrice l'idée empruntée à Jack Zipes de «subversion »; Nadine Jasmin a suivi davantage la méthode de Raymonde Robert, et à travers elle de Laurent Versini, en écartant l'interprétation littéraire pour s'attacher à décrire les phénomènes objectifs d'un corpus désormais assez bien connu, approche en outre assez marquée par Marc Fumaroli et par là tout opposée à Jean Mainil, et par l'idée, déjà avancée par Raymonde Robert, de la fonction mondaine et sociale du conte de fées. Marina Warner dit avoir surtout travaillé aux États-Unis, en Angleterre et en Hollande, et avoir bénéficié des lumières d'un des plus éminents des seiziémistes, Terence Cave. Sa démarche pourrait s'apparenter à celle que met en scène Fontenelle au début de l'essai De l'origine des fables : il y a quelque chose de stupéfiant dans les contes de fées, ils méritent d'être pris au sérieux. Pour cela, Marina Warner a choisi de mener une enquête sur les grandes largeurs. Elle porte sur l'ensemble du conte littéraire de l'antiquité à nos jours, en donnant une place privilégiée aux contes français de la fin du xvII siècle (elle ne s'intéresse presque pas aux contes des années 1730 et 1740 sinon pour La Belle et la Bête), et aux développements du genre aux $\mathrm{XIX}^{\mathrm{e}}$ et $\mathrm{XX}^{\mathrm{e}}$ siècles surtout en Angleterre (et aux 
États-Unis pour les textes plus récents). Mais elle veut en chemin montrer que l'on ne peut parler de ces textes qu'en considérant leurs conditions d'énonciation (ou de production) : le conte est porté par le conteur, ses rôles et ses images, et celui-ci résulte d'une histoire millénaire qui ne peut se découvrir qu'en remontant à ses figures telles que les textes et les documents iconographiques les plus variés permettent de les reconstituer.

Marina Warner dans une magistrale introduction, annonce que l'étude du conte de fées ne peut que s'appuyer sur l'histoire, parce que, pour reprendre des histoires qui circulent dans le temps et l'espace, le conte vaut par son rapport à un état donné de la société et à la situation concrète des individus : "le conte illumine des expériences engagées dans des conditions sociales et matérielles ». Mais le conte ne révèle pas seulement la diversité des appréhensions d'une réalité changeante, il touche plus directement le lecteur par ce qui fait son principe spécifique (ce que Jolles aurait appelé son "geste ») : il interroge les limites de l'identité et de la perception, telles qu'elle s'imposent à un moment de l'histoire humaine. Comme l'annonce le sous-titre, la compréhension de cette histoire se fait en un double mouvement : avant l'étude des figures du contenu (les contes), Marina Warner veut, en archéologue, retrouver les différentes figures qui, venues de très loin, ont fini par se fondre, ce qui explique qu'elles ne soient pas souvent reconnues comme telles dans la personne du conteur. Pour Marina Warner, cette figure est exclusivement féminine : dans le conte parle la voix des femmes (même si cette voix peut-être parlée par des auteurs comme Perrault, Grimm ou Andersen), et c'est essentiellement un discours sur les femmes et leur condition que le conte énonce. Ce qui unit les douze chapitres de la première partie (exactement la moitié du livre), et sert d'élément commun aux personnages historiques, mythiques ou littéraires successivement évoqués, est leur origine marginale, scandaleuse, inquiétante conférant en même temps l'accès à un savoir à la fois précieux et cryptique : cette position est celle le plus souvent de femmes élues qui sont, dans la transe ou le jeu, en communication avec un au-delà caché aux hommes. La mise à l'écart de la femme est ainsi la condition, sinon d'une assomption, du moins d'une sorte de vérité. Le récit de la vieille femme porte ainsi en lui le souvenir des Sybilles, de sainte Anne (la mère de Marie), de la reine de Saba, et par proximité, celui de l'âne de Balaam. Marina Warner se base sur les légendes ou les programmes iconographiques qui accompagnent chacune pour reconstituer les constellations d'associations qui accompagnent cette parole ambivalente, sale et précieuse : animaux symboliques (l'oie) ou tarés (le pied palmé de la reine de Saba), pratiques ludiques comme la devinette. On voit ainsi le conte de fées s'assimiler l'antiquité (l'âne d'or), et, au Moyen Âge, les légendes catholiques, la fiction arabe. Dans chaque cas, Marina Warner établit un lien étroit de cette figure féminine avec le conte de fées, et c'est surtout dans les deux derniers chapitres de cette partie qu'elle examine la figure de la conteuse sur laquelle ils se fondent, Basile pouvant à ce titre avoir rang d'initiateur. Elle voit ainsi le loup de Perrault absorber la grand-mère et le petit chaperon rouge comme lui-même s'assimile la conteuse populaire, tandis que les femmes écrivains ne peuvent si facilement jouer la part du sale et du bas: il leur faut en même temps affirmer leur distinction comme le montrent les Enchantements de l'éloquence de Lhéritier de Villandon.

La seconde partie du travail est consacrée aux contes, pour plus de la moitié les contes littéraires français, pour l'autre, essentiellement, des contes de langue anglaise, une 
place importante étant donnée à Angela Carter (morte en 1992). Se présentant comme un discours des femmes, riche de leur savoir propre et marqué par les diverses positions d'ignominie et d'effroi ou de vénération où elles sont placées, le conte de fées est donc ici lu principalement comme un discours sur la femme et pour les femmes: les différents chapitres vont s'organiser autour de personnages ou de rôles féminins récurrents dans les contes, ou de ceux à qui les femmes sont confrontées : de ce côté, les ogres, les amants démons (tous deux incarnés par Barbe bleue), les belles-mères coupables (avec La Belle au bois dormant), les mères absentes avec Cendrillon, de l'autre, les fiancées récalcitrantes ( $\mathrm{L} a$ Belle et la Bête), les femmes animales, les filles fugitives (Peau d'âne encore), les blondes, ou de façon plus abstraite, le langage des cheveux (Peau d'âne) et le silence filial (la petite ondine). Certains contes sont donc vus dans plusieurs perspectives au long de plusieurs chapitres: Peau d'âne et surtout La Belle et la Bête, la plus abondamment étudiée, et qui donne au volume son titre et pourrait-on dire l'une de ses lignes directrices. La démarche de Marina Warner est d'abord comparatiste: dans un va et vient constant entre littérature française et anglaise, dans la reconstitution des liens avec les traditions occidentales, mais aussi en considérant des versions parallèles des contes étudiés. Ainsi l'analyse de Cendrillon commence par raconter la version chinoise la plus ancienne de cette histoire ( $\mathrm{IX}^{\mathrm{e}}$ siècle), présente celle de Basile et utilise dans sa démonstration un conte camerounais. Démonstration, car le propos de Marina Warner est engagé : constatant la cruauté de la belle-mère dans ce conte (comme dans d'autres), elle remarque aussi que la lecture faite par Bettelheim autorise son accentuation dans les adaptations modernes (en partie celles de Walt Disney) au nom de l'ambivalence des sentiments des enfants pour les parents. Marina Warner se demande alors pourquoi un genre d'imprégnation féminine serait aussi hostile aux femmes? Ce doute l'amène à recourir aux leçons de l'histoire, c'est-à-dire du contexte réel de l'invention des contes. La mortalité des femmes qui accouchent est alors très forte, et les veufs se remarient presque tous immédiatement. Les familles recomposées et les conflits d'intérêts qu'elles entraînent sont donc un phénomène important. La belle-mère hostile n'est pas un fantasme, elle est un élément d'une situation. Ainsi s'explique le parcours opéré par Marina Warner dans son livre: il importe d'abord de considérer à quels sujets le conte est rapporté, par qui il est censé être dit, à qui il s'adresse. Dans le cas de Cendrillon elle remarque que le privilège propre du conteur est de dominer l'histoire et de préparer son issue heureuse peut prendre le visage féminin de la nourrice, la mie, la grand-mère ou la (belle) mère : à l'identification de l'enfant à l'héroïne persécutée, elle ajoute son identification à la figure parentale qui raconte le conte, et qui par sa seule présence corrige l'horreur des cruautés subies. Elle procède don à un déplacement concernant le contenu du conte en faisant le choix, contre une lecture immanente, d'une lecture qui tient compte des conditions pragmatiques de la transmission des contes, c'est-à-dire du sens que les auditeurs lecteurs, conteurs écrivains pouvaient donner au récit à partir de leur propre expérience: la propriété du conte de fées est qu'il inclut dans son écriture ces différentes "scènes", ou souvenirs de scènes, par lesquels il est passé. Processus continu jusqu'à aujourd'hui.

4 Il est impossible de rendre compte de l'extraordinaire richesse de chacun de ces onze chapitres, de la variété des références, de l'agilité des associations, de l'attention aux effets les plus fins, de la précision de l'information (les notes regroupées en fin de volume donnent toutes les références et indiquent l'étendue et la précision des lectures qui servent de support à ce livre). Le lecteur sera peut-être particulièrement sensible à 
l'intelligence du dossier iconographique: non seulement des images très variées et souvent très peu connues ou inconnues (en tout cas dans leur ensemble) mais surtout l'intégration des éléments qu'elles fournissent à l'analyse ou à l'enquête. Le lecteur sera peut-être plutôt particulièrement sensible à la présence de l'auteur, marquée d'humour et jamais hostile à son objet ou portée par un ressentiment contre le pouvoir des hommes, c'est elle qui assure à la fois le cheminement presque pédestre du texte et en même temps la clarté d'une direction ferme et toujours perspicace: restituer la figuration de la voix féminine, dégager les rôles féminins et le discours des écrivains, c'est mieux comprendre la spécificité et l'enjeu du conte. 